\title{
HER2-positive breast cancer that resists therapeutic drugs and ionizing radiation releases sphingomyelin-based molecules to circulating blood serum
}

\author{
SATORU MONZEN ${ }^{1}$, YOTA TATARA ${ }^{2}$, YASUSHI MARIYA ${ }^{3}$, MITSURU CHIBA $^{4}$, \\ ANDRZEJ WOJCIK ${ }^{5}$ and LOVISA LUNDHOLM ${ }^{5}$
}

\author{
${ }^{1}$ Department of Radiation Science, Hirosaki University Graduate School of Health Sciences, Hirosaki, Aomori 036-8564; \\ ${ }^{2}$ Department of Glycotechnology, Hirosaki University Graduate School of Medicine, Hirosaki, Aomori 036-8562; \\ ${ }^{3}$ Department of Radiology, Mutsu General Hospital, Mutsu, Aomori 035-0071; ${ }^{4}$ Department of \\ Bioscience and Laboratory Medicine, Hirosaki University Graduate School of Health Sciences, Hirosaki, \\ Aomori 036-8564, Japan; ${ }^{5}$ Centre for Radiation Protection Research, Department of Molecular Biosciences, \\ The Wenner-Gren Institute, Stockholm University, 11418 Stockholm, Sweden
}

Received February 17, 2020; Accepted June 16, 2020

DOI: $10.3892 / \mathrm{mco} .2020 .2140$

\begin{abstract}
Breast cancer is the second most common cancer in the world based on incidence, reaching more than 2 million new cases in 2018 , while continuing to increase. Invasive ductal carcinoma is the most common type of this cancer, making up approximately $70-80 \%$ of all breast cancer diagnoses. In particular, the type of breast cancer overexpressing human epidermal growth factor receptor 2 (HER2) has potential of strong proliferation, migration and invasion and early treatment is necessary. The authors identified and studied a single patient displaying complete therapeutic resistance to monoclonal anti-HER 2 antibody therapy, chemotherapy and radiotherapy. A patient who exhibited resistance to postoperative adjuvant therapy after mastectomy was selected from HER2-positive breast cancer, and this patient had the grade of $\mathrm{T}_{4 \mathrm{~b}} \mathrm{~N}_{2 \mathrm{a}} \mathrm{M}_{0}$, Stage IIIB. The patient samples, blood serum and cancer tissue, were analyzed by metabolome and immunostaining technique, respectively. The characteristics of peripheral blood serum and solid tumor were investigated, aiming to find new serum biomarker(s) using the metabolomics technique. A correlation between the appearance of HER2-positive cancer tissue and serum concentration of the sphingomyelin family was found. In addition, HER2-positive tumor tissue in both the primary and recurrent cancer express the sphingomyelinase. These results suggest that sphingomyelins from this cancer tissue leads to therapy resistance, induction of invasion and strong proliferation.
\end{abstract}

Correspondence to: Dr Satoru Monzen, Department of Radiation Science, Hirosaki University Graduate School of Health Sciences, 66-1 Hon-cho, Hirosaki, Aomori 036-8564, Japan

E-mail: monzens@hirosaki-u.ac.jp

Key wards: HER2-positive breast cancer, postoperative adjuvant therapy, resistant, metabolomics analysis, sphingomyeline

\section{Introduction}

Breast cancer is the second most common cancer worldwide with regard to incidence. Two million new cases were reached in 2018, a number that is continually on the increase (1). Invasive ductal carcinoma (IDC) is most common type, constituting 70-80\% of all breast cancer diagnoses (2). Abnormal cancer cells that form in the milk ducts spread beyond the ducts into other parts of the breast tissue (2). In particular, tumors with overexpressed or amplified human epidermal growth factor receptor 2 (HER2) show strong proliferation, migration and invasion. Early treatment is necessary, most commonly in the form of chemotherapy and surgery (3). Recently, HER2-directed targeted therapy using monoclonal antibodies, such as Trastuzumab and Pertuzumab, has been established as standard of care. Trastuzumab acts directly against the extracellular domain of the HER2 receptor and prevents ligand-independent HER2 signaling, downregulates HER2 expression and reduces the more active p95-HER2 form of HER2. Pertuzumab binds to the extracellular domain II of HER2 and inhibits ligand-dependent HER2-HER3 dimerization. These monoclonal antibody therapies are recommended for use together with radiotherapy and/or chemotherapy (4-8). However, some tumors are resistant to treatment by antibody therapy, chemotherapy and radiotherapy. The mechanisms of the resistance are not known.

In order to gain more insight into the resistance mechanisms, a metabolome analysis of serum collected repeatedly from a HER2-positive breast cancer patient who showed resistance to postoperative adjuvant therapy (PAT) was performed. The focus was on low-molecular-mass metabolites in blood because they, better than other 'omic' profiles, reflect the patients' biochemical status or physiopathological condition. Alterations at the metabolomic level not only reflect the alterations at the genomics and proteomics levels, but also are influenced by environmental factors (9).

In recent years, metabolomic studies have been successfully used to identify biomarkers and altered metabolic pathways in 
various cancer systems, including gastric, brain, breast, and lung cancer (10-13). In particular, a metabolomics approach was applied to identify biomarkers potentially associated with pathological complete response to trastuzumab-paclitaxel neoadjuvant therapy in HER2-positive breast cancer patients. Consequently, good responders showed higher levels of spermidine and lower amounts of tryptophan compared to the poor responders (14).

\section{Patients and methods}

Study subject and ethics approval. The PAT-resistant patient was selected from HER2-positive breast cancer patients (all $>40$ years) who were treated by mastectomy at the Mutsu General Hospital (Aomori, Japan) between February, 2017 and October, 2019. During this research period, a PAT-resistant case was found and further analysis was performed. The characteristic treatment flow and investigational points are summarized in Fig. 1A. The diagnosis was ductal adenocarcinoma in primary and solid tubular et scirrhous carcinoma in recurrent, $\mathrm{T}_{4 \mathrm{~b}} \mathrm{~N}_{2 \mathrm{a}} \mathrm{M}_{0}$, Stage IIIB. The patient was treated using Fluorouracil + Epirubicin Hydrochloride + Cyclophosphamide Hydrate (PAT1; day 18-78), and then with Trastuzumab + Paclitaxel (PAT2; day 102-466) (Fig. 1A) External radiotherapy (photon beam, 50 Gy per fraction, 25 fractions) was carried out on day 494-529 (PAT3). After surgery for removal of recurrent cancer tissue, the patient was treated using Trastuzumab + Pertuzumab + Docetaxel (PAT4; from day 669).

The study was approved by the Committee of Medical Ethics of the Hirosaki University Graduate School of Health Sciences (Hirosaki, Japan; no. 2016-051) and Mutsu General Hospital (Mutsu, Japan; no. 000-282) to ensure the welfare and privacy of the donors. Following a detailed verbal explanation regarding the content of this study, a written informed consent was obtained.

Follow up by blood test. To observe the patient's condition during the treatment, counts of white blood cells (WBC), red blood cells (RBC) and platelets (Plt) were recorded and the level of hemoglobin $(\mathrm{Hg})$ was measured by routine hematological techniques. At the same time, the concentration of C-reactive protein (CRP), carcinoembryonic antigen (CEA), cancer antigen 15-3 (CA15-3) and breast cancer antigen 225 (BCA225) were also quantified.

Collection of peripheral blood (PB) serum for metabolomics analysis. PB was collected directly into serum separation tubes (BD Biosciences). The serum was separated and stored at $-80^{\circ} \mathrm{C}$.

Mass spectrometry for metabolomics. Serum metabolites were measured as part of a targeted metabolomics panel using the AbsoluteIDQ P180 kit (Biocrates, Inc.). The kit is a fully automated assay based on phenylisothiocyanate (PITC) derivatization of the target analytes using internal standards for quantitation. Human serum sample preparation was carried out according to the manufacturer's protocol. Briefly, $10 \mu \mathrm{l}$ of serum was transferred to the first 96-well plate and dried under a nitrogen stream. Thereafter, $50 \mu 1$ of a $5 \%$ PITC solution was added to derivatize amino acids and biogenic amines. After incubation, the filter spots were dried again before the metabolites were extracted using $5 \mathrm{mM}$ ammonium acetate in methanol $(300 \mu \mathrm{l})$ into the second 96-well plate for analysis after further dilution using the Biocrates MS running solvent $\mathrm{A}$.

Acylcarnitines, lyso-phosphatidylcholines with acyl residue, phosphatidylcholine with diacyl residue sum (PC aa), and phosphatidylcholine with acyl-alkyl residue sum (PC ae), sphingomyelins, and the sum of hexoses were analyzed by the flow injection analysis (FIA) method in positive ion mode using the liquid chromatography-mass spectrometry (LC-MS/MS). The LC-MS/MS analysis was carried out by an HPLC system (ExionLC ${ }^{\text {TM }}$ AD, AB Sciex) coupled to a QTRAP6500+ triple quadruple ion trap hybrid mass spectrometer system (AB Sciex) in electrospray ionization (ESI) mode. The full list of metabolites and their abbreviations are presented in Table I. The metabolites were identified and quantified with multiple reaction monitoring (MRM) with individual transition and parameters of declustering potential (DP) and collision energy (CE) as listed in Table S1. Flow rate settings for FIA were as follows: $0.03 \mathrm{ml} / \mathrm{min}$ in $1.6 \mathrm{~min}$; 0.03 to $0.2 \mathrm{ml} / \mathrm{min}$ in $0.8 \mathrm{~min}$; $0.2 \mathrm{ml} / \mathrm{min}$ in $0.4 \mathrm{~min} ; 0.2$ to $0.03 \mathrm{ml} / \mathrm{min}$ in $0.2 \mathrm{~min}$. MS settings for FIA mode were as follows: Curtain gas, 45 ; ion spray voltage, $5,500 \mathrm{~V}$; temperature, $175^{\circ} \mathrm{C}$; ion source gas 1 , $40 \mathrm{psi}$; ion source gas 2, 50 psi; CAD gas, 6 psi; entrance potential, $10 \mathrm{~V}$; collision cell exit potential, $15 \mathrm{~V}$. Twenty microliters of the sample extract were used in the FIA. Quantification was carried out using internal standards and a calibration curve.

Amino acids and biogenic amines were analyzed via LC-MS/MS in positive ion mode. Then, $10 \mu \mathrm{l}$ of the sample extract were injected onto an HPLC C18 column (Zorbax Eclipse XDB-C18 column, 3x100 mm, $3.5 \mu \mathrm{m}$; Agilent) with a guard column (Zorbax SB-C18, 3x100 mm, $1.85 \mu \mathrm{m}$; Agilent) at $40^{\circ} \mathrm{C}$ using a 10 -min solvent gradient employing $0.2 \%$ formic acid in water (solvent $\mathrm{A}$ ) and $0.2 \%$ formic acid in acetonitrile (solvent B). Additional LC settings for LC-MS/MS were as follows: $0 \% \mathrm{~B}$ in $0.5 \mathrm{~min} ; 0-95 \% \mathrm{~B}$ in $5 \mathrm{~min} ; 95 \% \mathrm{~B}$ in $1 \mathrm{~min} ; 95-0 \% \mathrm{~B}$ in $0.5 \mathrm{~min} ; 0 \% \mathrm{~B}$ in $2.5 \mathrm{~min}$ at a flow rate of $0.5 \mathrm{ml} / \mathrm{min}$. MS settings for LC-MS/MS mode were as follows: Curtain gas, 45; ion spray voltage, $5,500 \mathrm{~V}$; temperature, $500^{\circ} \mathrm{C}$; ion source gas $1,40 \mathrm{psi}$; ion source gas $2,50 \mathrm{psi}$; CAD gas, 6 psi; entrance potential, $10 \mathrm{~V}$; collision cell exit potential, $15 \mathrm{~V}$. All metabolites were identified and quantified using isotopically-labeled internal standards and MRM as optimized and provided by Biocrates Inc.

Immunological analysis in HER2-positive cells in cancer tissue sections. Paraffin-embedded sections of the removed cancer tissues were deparaffinized with xylene and ethanol, washed in D-PBS (-) and treated with $3 \%$ hydrogen peroxide for $5 \mathrm{~min}$. The slides were treated with $10 \mathrm{mM}$ citrate buffer (pH 6.0) and incubated for $10 \mathrm{~min}$ at $121^{\circ} \mathrm{C}$ using an autoclave. Then, the slides were washed in TBS buffer $(25 \mathrm{mM}$ Tris- $\mathrm{HCl}$ and $150 \mathrm{mM} \mathrm{NaCl}, \mathrm{pH}$ 7.2). To perform blocking, the slides were treated with the blocking solution (5\% normal goat serum in TBS buffer) at room temperature for $30 \mathrm{~min}$. Next, the slides were incubated with the blocking solution containing a primary rabbit monoclonal antibody directed against human HER2/ERBB2 (no. 4290; Cell Signaling Technology Inc.) at a 1:1,000 dilution at room temperature for $60 \mathrm{~min}$. The slides 

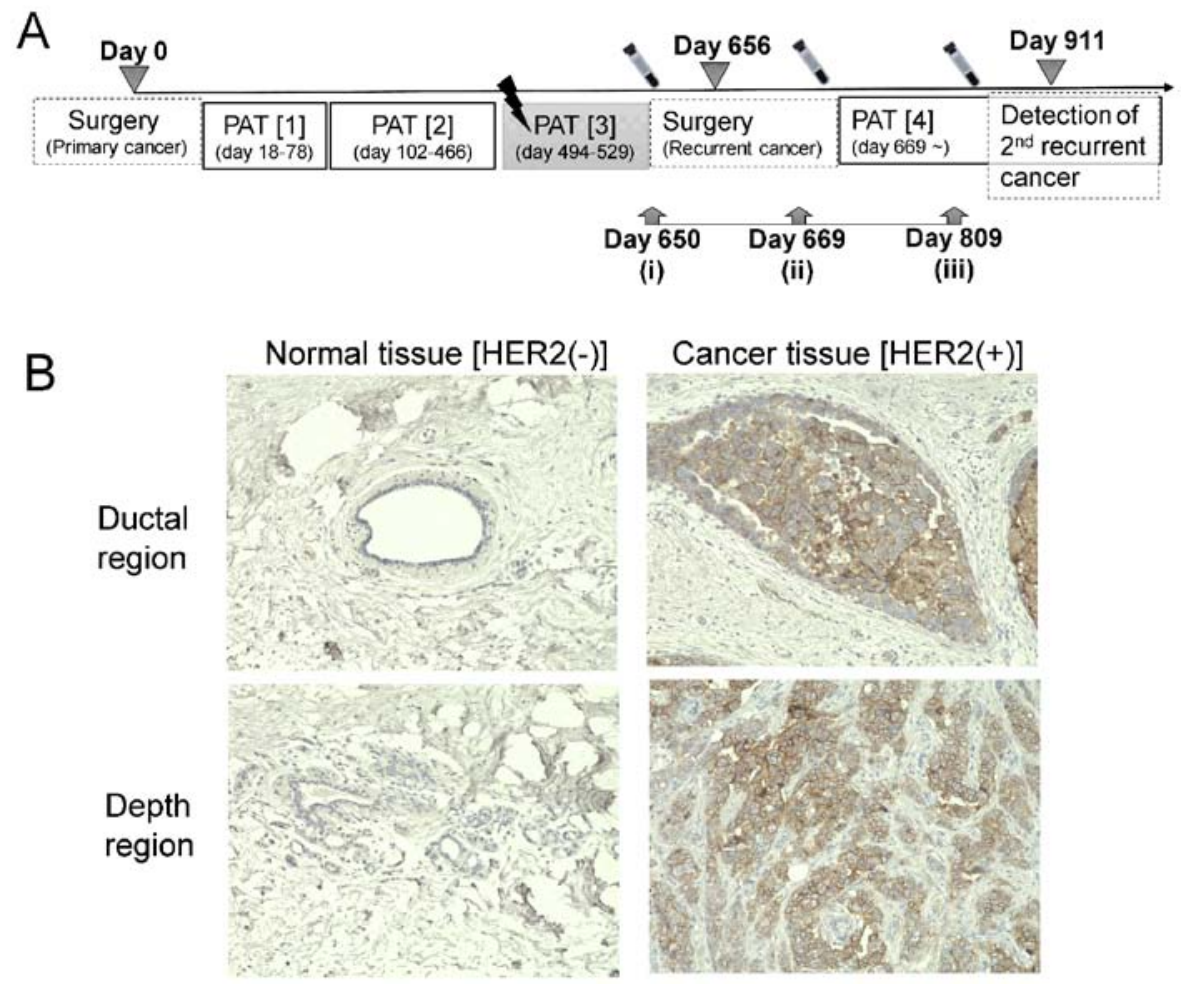

Figure 1. Treatment flow and HER2-expression. (A) Treatment flow of the studied HER2-expressing breast cancer patient. (B) Immunological staining of breast cancer tissue. Primary cancer tissue was removed by surgery immediately after diagnosis (day 0 ). The 1 st recurrent tumor tissue was removed on day 656 and 2nd recurrent tumor tissue was detected on day 911 (indicated by inverse triangles). The indicated days (650, i), (669, ii) and (809, iii) denote the collection of blood serum samples for metabolomic analysis. The administrated PATs were [1]: Fluorouracil + Epirubicin Hydrochloride + Cyclophospham ide Hydrate, [2]: Trastuzumab + Paclitaxel, [3]: External X-beam therapy (50 Gy in 25 daily fractions), [4]: Trastuzumab + Pertuzumab + Docetaxel. PAT, postoperative adjuvant therapy. The expression of HER 2 antigen in normal and tumor milk ductal tissues was analyzed in tissue samples removed on day 0. The depth regions of normal and tumor tissue were also analyzed.

were washed three times with TBS buffer and incubated at room temperature for $60 \mathrm{~min}$ with an anti-rabbit IgG horseradish peroxidase (HRP)-linked antibody (no. 7074, Cell Signaling Technology) prepared in the blocking solution at a 1:2,000 dilution. The slides were washed three times with TBS buffer at room temperature for $5 \mathrm{~min}$. Signals were visualized using 3,3'-diaminobenzidine (DAB) (Sigma-Aldrich). The slides were washed in water and nuclei were stained using hematoxylin solution. Finally, the slides were dehydrated with ethanol and xylene, and mounted using a hydrophobic mounting agent. Fluorescent immunological analysis was performed as in our previous study (15). Mouse monoclonal antibody directed against human nSMase2 (sc-166637; Santa Cruz Biotechnology, Inc.) (1:100 dilution) and rabbit monoclonal HER2/ERBB2 antibody (1:1,000 dilution) described above was used as a primary antibody. Anti-rabbit IgG Alexa Fluor 488-conjugated antibody (no. 4412, Cell Signaling Technology) and Anti-mouse IgG Alexa Fluor 647-conjugated antibody (no. 4410, Cell Signaling Technology) were used as secondary antibodies. The cell nuclear staining for fluorescence analysis DAPI (no. 4083; Cell Signaling Technology) was used. Fluorescence signals were detected using a fluorescence microscope (BZ-X700; Keyence).

\section{Results}

Patient description. Following diagnosis of right breast adenocarcinoma with the expression of HER 2 as primary cancer, the tumor was removed by surgery (day 0, Fig. 1A). Three months after completing PAT3 (day 606), locally recurrent cancer lesion in right breast within radiation field was detected and removed by surgery on day 656. A second locally recurrent cancer lesion and metastatic lymph node tumor were observed on day 911. According to pathological analysis, the removed primary tumor tissue (day 0) showed a distinctly higher expression of HER2 as compared to corresponding areas of normal tissue (Fig. 1B). A transient increase in WBC (days 1 and 657) and Plt (days 7-102 and 669) after surgery was observed (Fig. 2A and B). On the other hand, the number and concentration of RBC was stable (Fig. 2C and D). The inflammation marker CRP responded similarly to $\mathrm{WBC}$ and $\mathrm{Plt}$ (Fig. 2E), whereas the level of CEA remained stable (Fig. 2F). The levels of CA15-3 and BCA225 gradually increased during the observation period however these markers were within the normal range (Fig. $2 \mathrm{G}$ and $\mathrm{H}$ ).

Repeated tumor recurrence during PAT. After the first surgery, 4 cycles of PAT were performed (Fig. 1A). According to pathological analysis, the staging $\mathrm{T}_{1} \mathrm{~N}_{1} \mathrm{M} 0$ (Stage IIA) at the first diagnostic stage (before day 0 ) changed to $\mathrm{rT}_{4 \mathrm{~b}} \mathrm{~N}_{2 \mathrm{a}} \mathrm{M}_{0}$ when the recurrent tumor was detected in the same original region and with a part invading into the greater pectoral muscle as well as presenting as lymph node metastasis ( day 650$)$.

Metabolomic analysis in PB serum. None of the four PAT cycles showed effectiveness. To investigate whether specific 
Table I. The classification of metabolites in this study using the Biocrates AbsoluteIDQ ${ }^{\mathrm{TM}}$ kit.

\begin{tabular}{lll}
\hline Metabolite class & No. of analytes & Analyte name \\
\hline
\end{tabular}

Amino acid biogenic amine

42

Carnitine acylcarnitine

26

Hydroxy- and dicarboxyacylcarnitines

14

Sphingomyeline, hydroxysphingomyelins

Diacyl phosphatidylcholine

38

Acyl-alkyl phosphatidylcholine

38

Lysophosphatidylcholine

14

Sugar

Total

188
Ala, Arg, Asn, Asp, Cit, Gln, Glu, Gly, His, Ile, Leu, Lys, Met, Orn, Phe, Pro, Ser, Thr, Trp, Tyr, Val, Ac-Orn, ADMA, alpha-AAA, c4-OH-Pro, Carnosine, Creatinine, DOPA, Dopamine, Histamine, Kynurenine, Met-SO, Nitro-Tyr, PEA, Putrescine, SDMA, Serotonin, Spermidine, Spermine, t4-OH-Pro, Taurine, total DMA C0, C2, C3, C3:1, C4, C4:1, C5, C5:1, C6 (C4:1-DC), C6:1, C8, C9, C10, C10:1, C10:2, C12, C12:1, C14, C14:1, C14:2, C16, $\mathrm{C} 16: 1, \mathrm{C} 16: 2, \mathrm{C} 18, \mathrm{C} 18: 1, \mathrm{C} 18: 2$

C3-DC (C4-OH), C3-OH, C5-DC (C6-OH), C5-M-DC, C5-OH (C3-DC-M), C5:1-DC, C7-DC, C12-DC, C14:1-OH, C14:2-OH, C16-OH, C16:1-OH, C16:2-OH, C18:1-OH

$\mathrm{SM}(\mathrm{OH}) \mathrm{C} 14: 1, \mathrm{SM}(\mathrm{OH}) \mathrm{C} 16: 1, \mathrm{SM}(\mathrm{OH}) \mathrm{C} 22: 1, \mathrm{SM}(\mathrm{OH})$ C22:2, SM (OH) C24:1, SM C16:0, SM C16:1, SM C18:0, SM C18:1, SM C20:2, SM C22:3, SM C24:0, SM C24:1, SM C26:0, SM C26:1

PC aa C24:0, PC aa C26:0, PC aa C28:1, PC aa C30:0, PC aa $\mathrm{C} 30: 2, \mathrm{PC}$ aa C32:0, PC aa C32:1, PC aa C32:2, PC aa C32:3, PC aa C34:1, PC aa C34:2, PC aa C34:3, PC aa C34:4, PC aa C36:0, $\mathrm{PC}$ aa $\mathrm{C} 36: 1, \mathrm{PC}$ aa $\mathrm{C} 36: 2, \mathrm{PC}$ aa $\mathrm{C} 36: 3, \mathrm{PC}$ aa $\mathrm{C} 36: 4, \mathrm{PC}$ aa C36:5, PC aa C36:6, PC aa C38:0, PC aa C38:1, PC aa C38:3, PC aa C38:4, PC aa C38:5, PC aa C38:6, PC aa C40:1, PC aa C40:2, PC aa C40:3, PC aa C40:4, PC aa C40:5, PC aa C40:6, PC aa C42:0, PC aa C42:1, PC aa C42:2, PC aa C42:4, PC aa C42:5, PC aa C42:6 $\mathrm{PC}$ ae $\mathrm{C} 30: 0, \mathrm{PC}$ ae $\mathrm{C} 30: 1, \mathrm{PC}$ ae $\mathrm{C} 30: 2$, PC ae $\mathrm{C} 32: 1$, PC ae C32:2, PC ae C34:0, PC ae C34:1, PC ae C34:2, PC ae C34:3, PC ae C36:0, PC ae C36:1, PC ae C36:2, PC ae C36:3, PC ae C36:4, $\mathrm{PC}$ ae $\mathrm{C} 36: 5, \mathrm{PC}$ ae $\mathrm{C} 38: 0, \mathrm{PC}$ ae $\mathrm{C} 38: 1, \mathrm{PC}$ ae $\mathrm{C} 38: 2$, PC ae C38:3, PC ae C38:4, PC ae C38:5, PC ae C38:6, PC ae C40:1, PC ae $\mathrm{C} 40: 2$, PC ae $\mathrm{C} 40: 3$, PC ae $\mathrm{C} 40: 4, \mathrm{PC}$ ae $\mathrm{C} 40: 5$, PC ae $\mathrm{C} 40: 6$, $\mathrm{PC}$ ae $\mathrm{C} 42: 0, \mathrm{PC}$ ae $\mathrm{C} 42: 1, \mathrm{PC}$ ae $\mathrm{C} 42: 2$, PC ae $\mathrm{C} 42: 3$, PC ae $\mathrm{C} 42: 4$, PC ae C42:5, PC ae C44:3, PC ae C44:4, PC ae C44:5, PC ae C44:6 lysoPC a C14:0, lysoPC a C16:0, lysoPC a C16:1, lysoPC a C17:0, lysoPC a C18:0, lysoPC a C18:1, lysoPC a C18:2, lysoPC a C20:3, lysoPC a C20:4, lysoPC a C24:0, lysoPC a C26:0, lysoPC a C26:1, lysoPC a C28:0, lysoPC a C28:1

$\mathrm{H} 1$

Cx:y (x, number of carbons in the fatty acid side chain; $y$, number of double bonds in the fatty acid side chain); DC, decarboxyl; OH, hydroxyl; M, methyl; PC, phosphatidylcholine; aa, acyl-acyl; ae, acyl-alkyl; a, lyso; SM, sphingomyelin.

biomarker(s) were released from cancer tissues to circulating body fluids, PB serum was collected at the time before surgery for 1 st recurrent tissue (day 650, Fig. 1-i), after 13 days from surgery (day 669, Fig. 1ii) and the day when the 2nd recurrent cancer was diagnosed (day 809, Fig. 1-iii), and analyzed by mass spectrometry for wide-targeted metabolomic markers of response. The concentration of 188 molecules was analyzed (Table I), and, of these, 40 upregulated molecules and 28 downregulated molecules were observed on day 669 (Fig. 1A-ii) as compared to day 650 (Fig. 1A-i) (Fig. 3A and B) by merely calculating the molecules in Table I. In addition, 23 molecules among the 40 upregulated molecules (Fig. 1A-ii) were downregulated on day 809 (Fig. 1A-iii) in comparison to day 669 (Fig. 1A-ii). On the other hand, 23 molecules of the 28 downregulated molecules on day 669 (as compared to day 650) were upregulated on day 809 (as compared to day 669). These molecules that were up-to-down- and down-to-up-regulated contained many lipids of three classes: Acyl-alkyl phosphatidylcholine (6 molecules), sphingomyeline (4 molecules) and lysophosphatidylcholine (lysoPC) (7 molecules) (Fig. 3C). Interestingly, the concentrations of molecules in these three 
A WBC
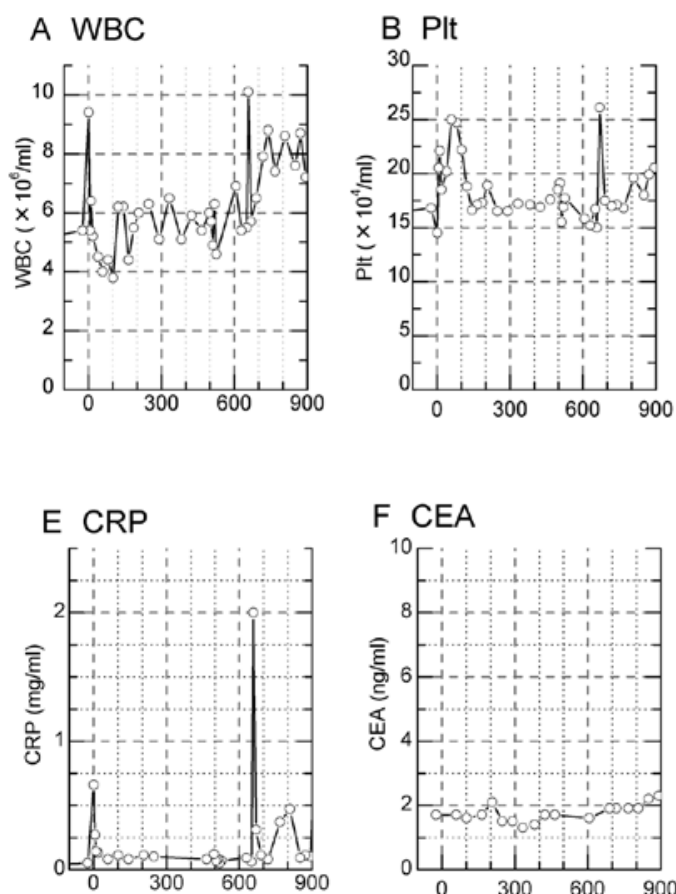

C RBC

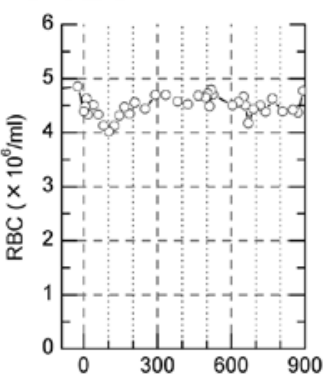

F CEA

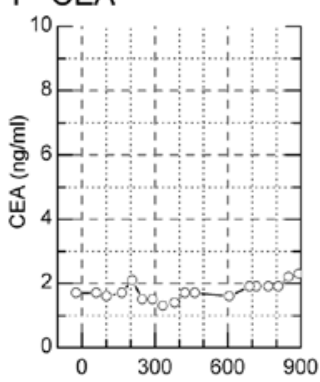

G CA15-3

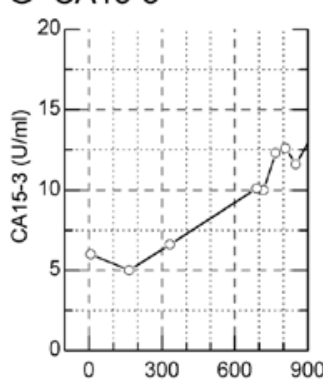

$\mathrm{D} \mathrm{Hb}$

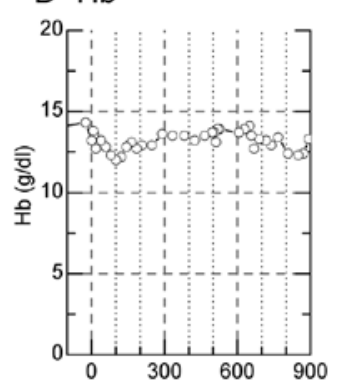

H BCA225

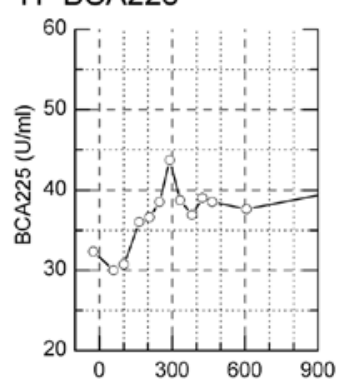

Period from surgery of primary cancer (days)

Figure 2. Peripheral blood cell counts, haemoglobin, and biochemical markers during the course of therapy. Concentrations of (A) white blood cell (WBC), (B) platelet (Plt), (C) red blood cell (RBC), (D) level of hemoglobine (Hb), (E) C-reactive protein (CRP) inflammation marker, and (F, G and H) breast cancer-specific markers carcinoembryonic antigen (CEA), cancer antigen 15-3 (CA15-3) and breast cancer antigen 225 (BCA225), respectively. The normal range of WBC, Plt, RBC, Hb, CRP, CEA, CA15-3 and BCA225 were: $4.5-11.0 \times 10^{6} / \mathrm{ml}, 1.5-4.5 \times 10^{5} / \mathrm{ml}, 4.2-5.4 \times 10^{6} / \mathrm{ml}, 12.5-15.5 \mathrm{~g} / \mathrm{dl},<3.0 \mathrm{mg} / \mathrm{ml},<5.0 \mathrm{ng} / \mathrm{ml}$, $30 \mathrm{U} / \mathrm{ml}$ and $<160 \mathrm{U} / \mathrm{ml}$, respectively.

classes showed fluctuations which correlated with the detection of cancer recurrence (Fig. 3C). LysoPC is produced from partial hydrolysis of phosphatidylcholines, and is regarded as an inflammation-resolution lipid mediator (16). In this case study, half of the 14 lysoPC species measured were increased after surgery and decreased when cancer recurred.

Immunoexpression analysis in cancer tissue. Phosphatidylcholines and sphingomyelins are known as components of cell membranes, especially in extracellular vesicles which are associated with cell-to-cell communication (17-19). Therefore, we focused on neutral sphingomyelinase-2 (nSMase), which is one of the important factors for exocytosis or endocytosis. In primary cancer tissue and 1st recurrent cancer tissue, a higher expression of Her2 protein was observed (Fig. 4). However, the lowest accumulation of intracellular nSMase in recurrent tumor was shown in comparison to normal or primary tumor. This means that the exocytosis or endocytosis occurs for release as extracellular vesicles (EVs).

\section{Discussion}

In the present study, we focused on a single HER2-positive breast cancer patient with PAT resistance to identify the characteristics of serum metabolites using metabolome analysis. The applied PATs were not effective in their anti-tumorigenic, anti-invasion and anti-lymph node metastatic action. In addition, the biochemical markers in peripheral blood did not respond to the PATs in a strong manner. It is known that $\sim 60 \%$ of patients with HER2-positive breast cancer develop resistance to trastuzumab, partially due to loss of expression of HER2 extracellular domain on their tumor cells (20). In clinical sites, this is sometimes diagnosed as a resistant fraction to administration of chemotherapeutic drugs (i.e., taxans) and/or external photon radiotherapy in HER2-positive cancer patients. However, the essential cause or the key factor driving this resistance remains to be determined. Previous findings have shown that the PI3K/Akt/mTOR pathway, STAT3-survivin signaling and expression of mitogen-activated protein kinase phosphatase 1 produce for an environment that promotes resistance (21-23). Therefore, there may be different subpopulations of cancer cells that are responsive or non-responsive to cytotoxic drugs or ionizing radiation in HER2-positive breast cancer.

A poor response of traditional breast cancer biomarkers in serum (CEA, CA15-3 and BCA225) was observed in the blood of the studied patient (Fig. 2) and they were of no value for predicting the recurrence of cancer until the pathological test was performed and diagnosed with correctly (day 645-650). Therefore, the identification of earlier specific biomarkers of HER2-positive breast cancer progression and therapeutic effectiveness is needed. We aimed to identify new serum biomarker(s) using the metabolomics technique and found that the concentration of the sphingomyeline family in serum is inverse-correlated to the accumulation of nSMase in HER2-positive cancer tissue (Figs. 3 and 4). Sphingomyelin is one of the sphingolipids, an important structural component of biological membranes and one of the end-points in the synthesis of sphingolipids (24). Its hydrolysis releases ceramide 
A

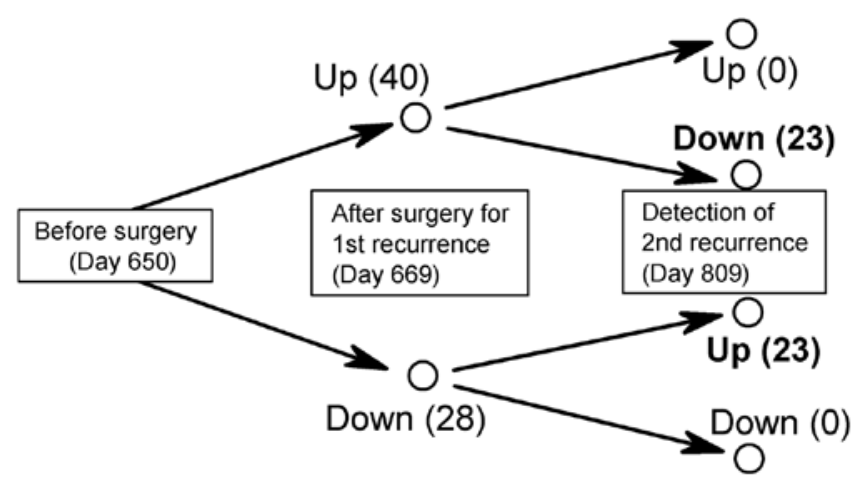

B

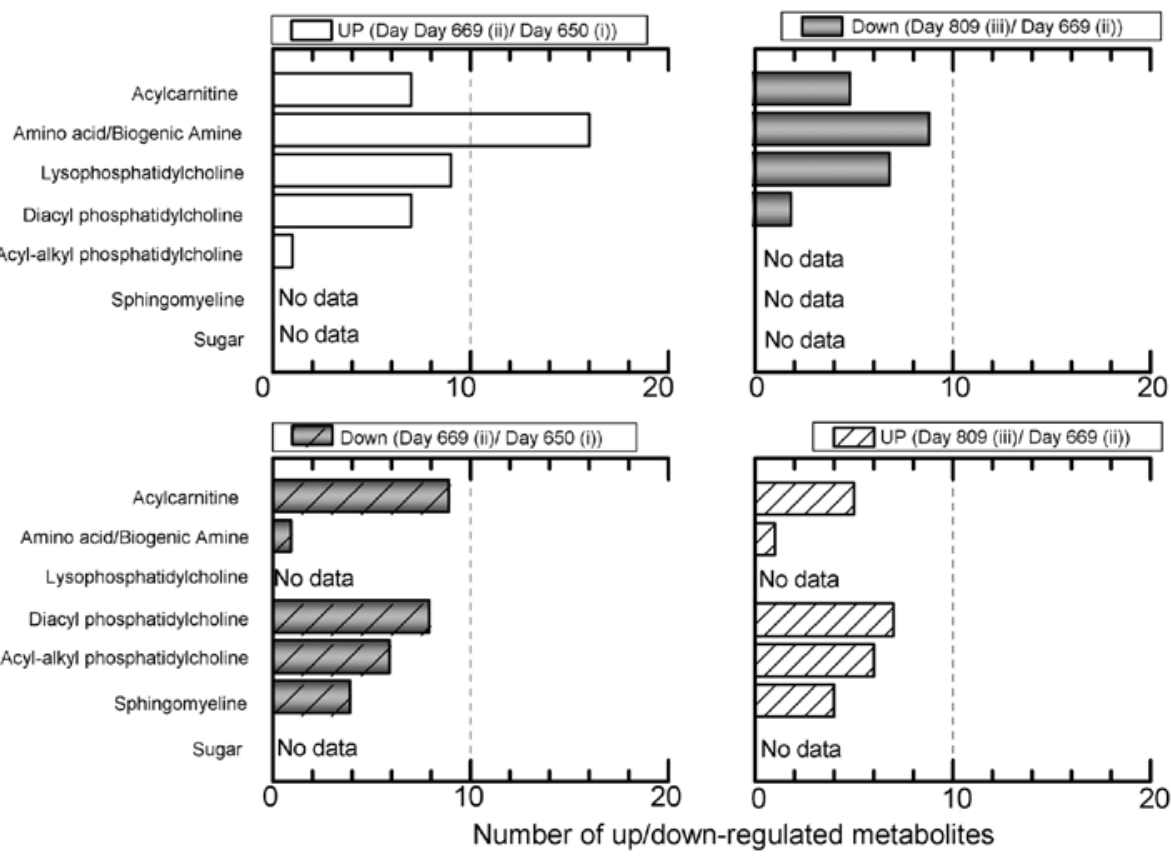

C
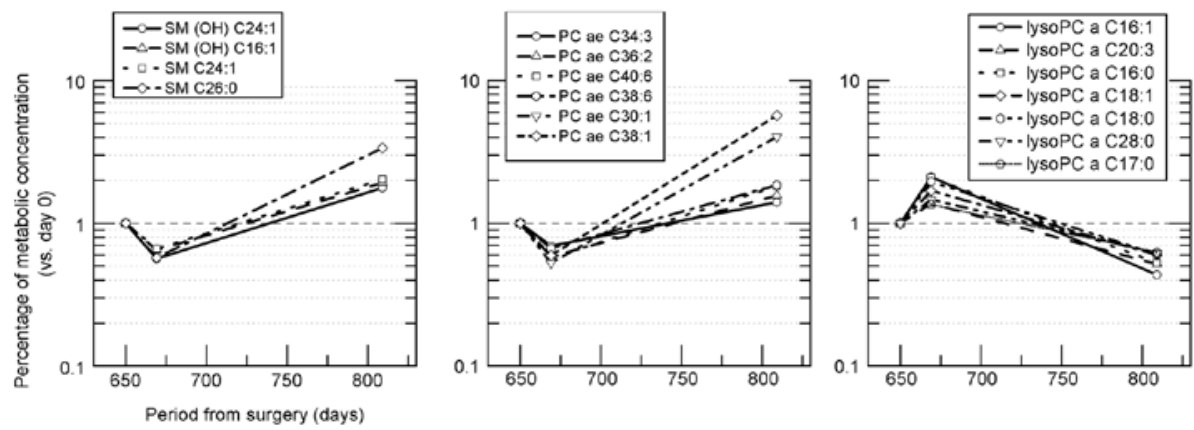

Figure 3. Serum metabolites in blood samples collected on days 650,669 and 809 (further details in Fig. 1); 1.3-fold up-regulated (Up) and 0.7-fold down-regulated (Down) molecules were extracted. (A) Overall map of the number of regulated metabolite molecules at different time points. (B) The molecules were classified into seven components. (C) The detailed molecules in the classes 'Sphingomyelin', 'Acyl-alkyl phosphatidylcholine', and 'LysoPC' were specified.

and phosphocholine and several stimuli are known to activate sphingomyelin hydrolysis (25). Ceramide is an important promoter of apoptosis (18), but additionally, ceramide also triggers budding of EVs, such as exosomes into multivesicular endosomes (26). It is known that EVs are nanometer-scale particles that are secreted by cells and mediate intercellular communication by transferring biomolecules between cells. Thus, their expression relates to cancer tissue growth or distant metastasis. We have previously reported that during the active release of EVs to blood flow after exposure to higher dose of ionizing radiation, the lower accumulation of EV components in their original tissue cells was observed in comparison to the other tissues with non-active release of EVs $(27,28)$. Our case data showed that the concentration of nSMase co-expressed with HER2 in 1st recurrent cancer tissue, where the levels of both are lower than in primary cancer tissue (Fig. 4), and a 

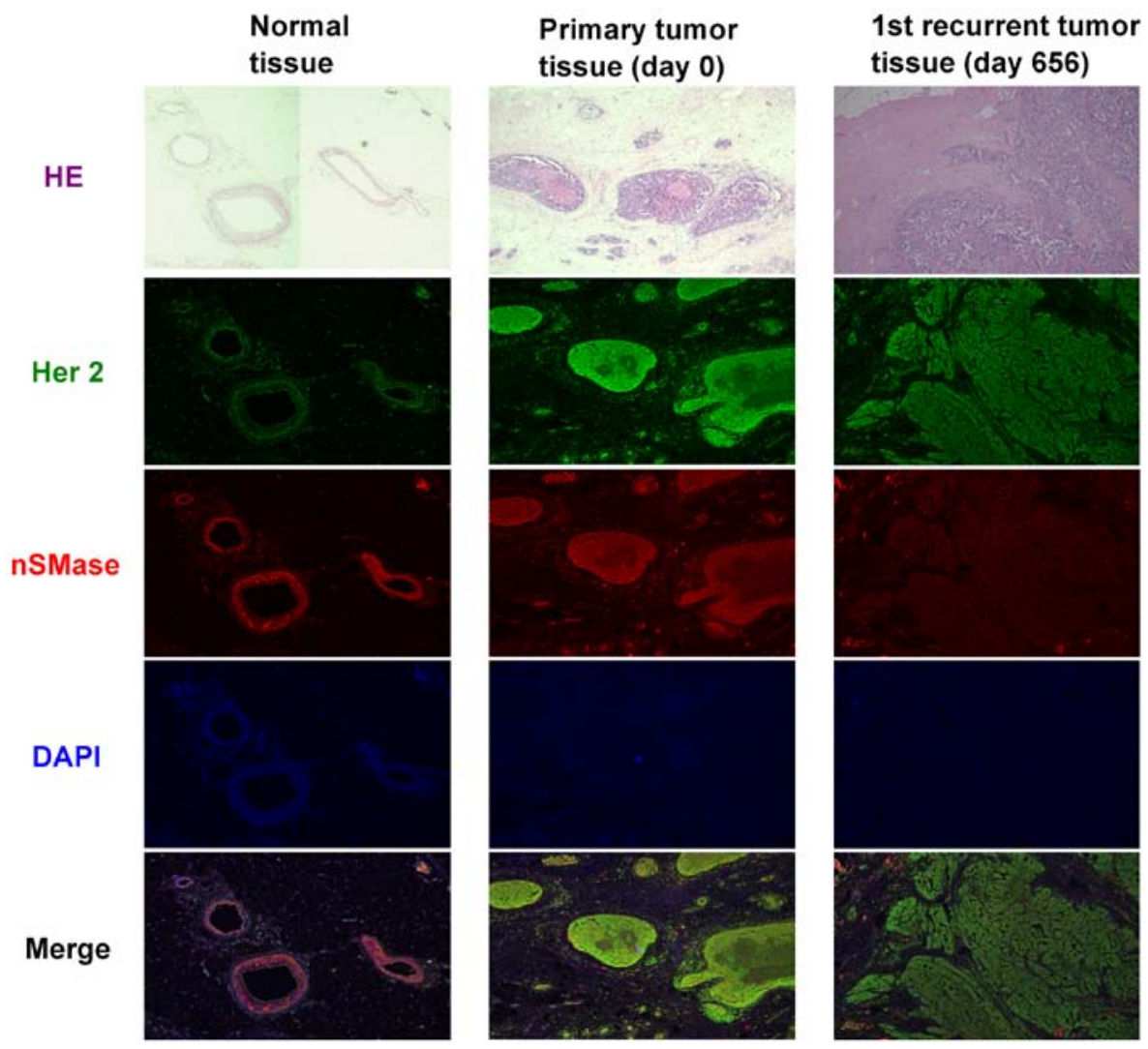

Figure 4. Identification of hematoxylin and eosin staining tissue (H\&E), HER2-positive tissue (green) and nSMase-positive tissue (red) in surgically removed breast cancer tissues. DAPI (blue) staining that is able to identify the cell nuclear and merged images are also shown.

higher concentration of sphingomyelin family in serum was detected when the 2 nd recurrent cancer tissue was diagnosed. These responses suggested that recurrent cancer tissues more easily release EVs to peripheral blood serum and promote cancer progression and expansion. Thus, one reported mechanism is the transfer of miRNAs via exosomes from cancer cells to microenvironmental cells, promoting angiogenesis $(28,29)$.

It is possible that full resistance to PAT could be connected to sphingomyelines, which may serve as a serum marker. The gene sphingomyeline phosphodiesterase 3 (SMPD3) which codes for nSMase was shown to act as a tumor suppressor gene in hepatocellular carcinoma, supported by overexpression and knockdown experiments (30). Of note, a recent study showed that high levels of sphingomyelin synthase 2 (SGMS2), which controls the synthesis of sphingomyelins from ceramide and thereby disrupts the ceramide-associated apoptosis pathway, is correlated with breast cancer aggressiveness and metastasis (31).

Additionally, we saw a clear opposite pattern for diacyl and acyl-alkyl phosphatidylcholines, where there was a decrease at 30 days after surgery, and then an increase, versus lysoPCs, which was initially increased and then decreased. Histological damage and infection caused by surgery induce inflammatory biological responses which are evoked as a result of exogenous and endogenous mediators secreted not only locally but also systemically. Of note is that increased levels of the enzyme lysoPC acyltransferase 1 (LPCAT1), controlling the conversion of lysoPC to phosphatidylcholine, were linked to poor prognosis and adverse features such as estrogen and progesterone receptor negativity, amplification of HER2 and MYC and deletions of PTEN and CDKNA2 in breast cancer $(32,33)$. Kim et al also reported a similar statement that lipid profiles, such as sphingomyelin and phosphatidylcholine, correlated with various clinicopathological characteristics of HER2-positive breast cancer (34).

Although the PAT resistance could not be clearly deciphered, our results provide novel information of a parallel expression intensity of HER 2 and nSMase, which possibly is connected to cell communication by EVs. Recently, alteration of gene expression by EV transportation was reported to induce trastuzumab-resistant HER2-positive metastatic breast cancer (35). Clearly, additional, detailed analysis is required on a larger number of patients.

In conclusion, complete therapeutic-resistant cancer tissue connected to reduced expression of nSMase in HER2-positive breast cancer are strongly co-expressed with the sphingomyelin family which can be detected at the peripheral blood serum level.

\section{Acknowledgements}

The authors are grateful to Miyu Miyazaki at the Center for Scientific Equipment Management, Hirosaki University Graduate School of Medicine, for assistance with LC-MS/MS analysis.

\section{Funding}

This study was supported by 'JSPS KAKENHI, Grant-in-Aid for Scientific Research (C) (General) (project no. 16K10339, 
Satoru Monzen)', 'JSPS KAKENHI, Fund for the Promotion of Joint International Research (Fostering Joint International Research) (project no. 17KK0181, Satoru Monzen)', and 'Interdisciplinary Collaborative Research Grant for Young Scientists, Hirosaki University'.

\section{Availability of data and materials}

All data generated or analysed during the present study are included in this published article.

\section{Authors' contributions}

SM and YM designed the study and prepared the manuscript draft and substantively participated in revising the manuscript. SM, YT and MC conceived the study, and contributed by analyzing the patient's data and revised the manuscript. AW and LL supervised the study and critically reviewed the manuscript, and gave final approval of the version to be published. All authors read and approved the final manuscript.

\section{Ethics approval and consent to participate}

The study was approved by the Committee of Medical Ethics of the Hirosaki University Graduate School of Health Sciences, (no. 2016-051) and Mutsu General Hospital (no. 000-282) to ensure the welfare and privacy of the donors. Following a detailed verbal explanation regarding the content of this study, a written informed consent was obtained.

\section{Patient consent for publication}

Not applicable.

\section{Competing interests}

The authors declare that they have no competing interests.

\section{References}

1. Bray F, Ferlay J, Soerjomataram I, Siegel RL, Torre LA and Jemal A: Global cancer statistics 2018: GLOBOCAN estimates of incidence and mortality worldwide for 36 cancers in 185 countries. CA Cancer J Clin 68: 394-424, 2018.

2. Tsuchiya SI, Yamaguchi R, Tsuchiya $K$ and Ohashi R: Characteristics of the Japanese histological classification for breast cancer: Correlations with imaging and cytology. Breast Cancer 23: 534-539, 2016.

3. Ahmed S, Sami A and Xiang J: HER2-Directed therapy: Current treatment options for HER2-positive breast cancer. Breast Cancer 22: 101-116, 2015.

4. Wuerstlein R and Harbeck N: Neoadjuvant therapy for HER2-positive breast cancer. Rev Recent Clin Trials 12: 81-92, 2017.

5. Kubo M, Kawai M, Kumamaru H, Miyata H, Tamura K, Yoshida M, Ogo E, Nagahashi M, Asaga S, Kojima Y, et al: A population-based recurrence risk management study of patients with pT1 node-negative HER $2^{+}$breast cancer: A national clinical database study. Breast Cancer Res Treat 178: 647-656, 2019.

6. Goldvaser H, Korzets Y, Shepshelovich D, Yerushalmi R, Sarfaty M, Ribnikar D, Thavendiranathan P and Amir E: Deescalating adjuvant trastuzumab in HER2-positive early-stage breast cancer: A systemic review and meta-analysis. JNCI Cancer Spectr 3: pkz033, 2019.
7. Hirsova P, Ibrahim SH, Krishnan A, Verma VK, Bronk SF, Werneburg NW, Charlton MR, Shah VH, Malhi H and Gores GJ: Lipid-Induced signaling causes release of inflammatory extracellular vesicles from hepatocytes. Gastroenterology 150: 956-967, 2016.

8. Gu G, Dustin D and Fuqua SA: Targeted therapy for breast cancer and molecular mechanisms of resistance to treatment. Curr Opin Pharmacol 31: 97-103, 2016.

9. Gomez-Casati DF and Busi MV: Clinical Molecular Medicine. In: Principles and Practice. 1st Edition. Kumar D (ed.) Academic Press, pp47-55, 2019.

10. Wang D, Li W, Zou Q, Yin L, Du Y, Gu J and Suo J: Serum metabolomic profiling of human gastric cancer and its relationship with the prognosis. Oncotarget 8: 110000-110015, 2017.

11. Chinnaiyan P, Kensicki E, Bloom G, Prabhu A, Sarcar B, Kahali S, Eschrich S, Qu X, Forsyth P and Gillies R: The metabolomic signature of malignant glioma reflects accelerated anabolic metabolism. Cancer Res 72: 5878-5888, 2012.

12. Hadi NI, Jamal Q, Iqbal A, Shaikh F, Somroo S and Musharraf SG: Serum metabolomic profiles for breast cancer diagnosis, grading and staging by gas chromatography-mass spectrometry. Sci Rep 7: 1715, 2017.

13. Kumar N, Shahjaman M, Mollah MN, Islam SM and Hoque MA: Serum and plasma metabolomic biomarkers for lung cancer. Bioinformation 13: 202-208, 2017.

14. Miolo G, Muraro E, Caruso D, Crivellari D, Ash A, Scalone S, Lombardi D, Rizzolio F, Giordano A and Corona G: Pharmacometabolomics study identifies circulating spermidine and tryptophan as potential biomarkers associated with the complete pathological response to trastuzumab-paclitaxel neoadjuvant therapy in HER-2 positive breast cancer. Oncotarget 7: 39809-39822, 2016.

15. Chiba M, Kubota S, Sakai A and Monzen S: Cell-To-Cell communication via extracellular vesicles among human pancreatic cancer cells derived from the same patient. Mol Med Rep 18: 3989-3996, 2018.

16. Yan JJ, Jung JS, Lee JE, Lee J, Huh SO, Kim HS, Jung KC, Cho JY, Nam JS, Suh HW, et al: Therapeutic effects of lysophosphatidylcholine in experimental sepsis. Nat Med 10: 161-167, 2004.

17. van Meer G, Voelker DR and Feigenson GW: Membrane lipids: Where they are and how they behave. Nat Rev Mol Cell Biol 9: $112-124,2008$

18. Shamseddine AA, Airola MV and Hannun YA: Roles and regulation of neutral sphingomyelinase-2 in cellular and pathological processes. Adv Biol Regul 57: 24-41, 2015.

19. Andrews NW, Corrotte M and Castro-Gomes T: Above the fray: Surface remodeling by secreted lysosomal enzymes leads to endocytosis-mediated plasma membrane repair. Semin Cell Dev Biol 45: 10-17, 2015.

20. Nami B and Wang Z: HER2 in breast cancer stemness: A negative feedback loop towards trastuzumab resistance. Cancers (Basel) 26: 40, 2017.

21. Wilks ST: Potential of overcoming resistance to HER2-targeted therapies through the PI3K/Akt/mTOR pathway. Breast 24: 548-555, 2015.

22. Kim JS, Kim HA, Seong MK, Seol H, Oh JS, Kim EK, Chang JW, Hwang SG and Noh WC: STAT3-Survivin signaling mediates a poor response to radiotherapy in HER2-positive breast cancers. Oncotarget 7: 7055-7065, 2016.

23. Candas D and Li JJ: MKP1 mediates resistance to therapy in HER2-positive breast tumors. Mol Cell Oncol 2: e997518, 2015.

24. Adada M, Luberto $C$ and Canals D: Inhibitors of the sphingomyelin cycle: Sphingomyelin synthases and sphingomyelinases. Chem Phys Lipids 197: 45-59, 2016.

25. Signorelli P and Hannun YA: Analysis and quantitation of ceramide. Methods Enzymol 345: 275-294, 2002.

26. Elsherbini A and Bieberich E: Ceramide and exosomes: A novel target in cancer biology and therapy. Adv Cancer Res 140: 121-154, 2018.

27. Chiba M, Kubota S, Sato K and Monzen S: Exosomes released from pancreatic cancer cells enhance angiogenic activities via dynamin-dependent endocytosis in endothelial cells in vitro. Sci Rep 8: 11972, 2018.

28. Chiba M, Monzen S, Iwaya C, Kashiwagi Y, Yamada S, Hosokawa Y, Mariya Y, Nakamura T and Wojcik A: Serum miR-375-3p increase in mice exposed to a high dose of ionizing radiation. Sci Rep 8: 1302, 2018. 
29. Kosaka N, Iguchi H, Hagiwara K, Yoshioka Y, Takeshita F and Ochiya T: Neutral sphingomyelinase 2 (nSMase2)-dependent exosomal transfer of angiogenic microRNAs regulate cancer cell metastasis. J Biol Chem 288: 10849-10859, 2013.

30. Revill K, Wang T, Lachenmayer A, Kojima K, Harrington A, Li J, Hoshida Y, Llovet JM and Powers S: Genome-Wide methylation analysis and epigenetic unmasking identify tumor suppressor genes in hepatocellular carcinoma. Gastroenterology 145: 1424-1435, 2013

31. Zheng K, Chen Z, Feng H, Chen Y, Zhang C, Yu J, Luo Y, Zhao L, Jiang $X$ and Shi F: Sphingomyelin synthase 2 promotes an aggressive breast cancer phenotype by disrupting the homoeostasis of ceramide and sphingomyelin. Cell Death Dis 10: 157, 2019.

32. Lebok P, von Hassel A, Meiners J, Hube-Magg C, Simon R, Höflmayer D, Hinsch A, Dum D, Fraune C, Göbel C, et al: Up-regulation of lysophosphatidylcholine acyltransferase 1 (LPCAT1) is linked to poor prognosis in breast cancer. Aging (Albany NY) 11: 7796-7804, 2019.
33. Abdelzaher E and Mostafa MF: Lysophosphatidylcholine acyltransferase 1 (LPCAT1) upregulation in breast carcinoma contributes to tumor progression and predicts early tumor recurrence. Tumor Biol 36: 5473-5483, 2015.

34. Kim IC, Lee JH, Bang G, Choi SH, Kim YH, Kim KP, Kim HK and Ro J: Lipid profiles for HER2-positive breast cancer. Anticancer Res 33: 2467-2472, 2013.

35. de Oliveira Taveira M, Nabavi S, Wang Y, Tonellato P, Esteva FJ, Cantley LC and Wulf GM: Genomic characteristics of trastuzumab-resistant her2-positive metastatic breast cancer. J Cancer Res Clin Oncol 143: 1255-1262, 2017.

(i) (8) This work is licensed under a Creative Commons Attribution-NonCommercial-NoDerivatives 4.0 International (CC BY-NC-ND 4.0) License. 